\title{
Research Paper \\ Investigating the Relationship Between Human Temperament and Environmental Color: A Cross-Sectional-Analytical Study in Kerman City (2017)
}

\author{
* Vahideh Rahimi Mehr ${ }^{1}$ \\ 1. Department of Architecture, Faculty of Art, Architecture \& Urbanism, Kerman Branch, Islamic Azad University, Kerman, Iran
}

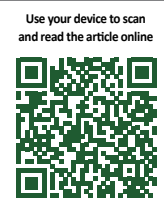

Crtation: Rahimi Mehr V. [Investigating the Relationship Between Human Temperament and Environmental Color: A CrossSectional-Analytical Study in Kerman City (2017) (Persian)]. Complementary Medicine Journal. 2020; 10(2):160-173. https:// doi.org/10.32598/cmja.10.2.970.1

doil'https://doi.org/10.32598/cmja.10.2.970.1

Key words: Color, Architecture, Traditional Persian Medicine, Temperament

\section{ABSTRACT}

Objective Environmental factors can affect the brain and affect other organs through the brain. Color and light are among the environmental factors that are considered the main features of architecture and they are more in our control. According to traditional medicine theories, the Temperament of a person and his health is affected by various factors. Color is one of the factors affecting health that has not been investigated by researchers so far, so the purpose of this study was to investigate the relationship between the color of the environment as an external factor and the Temperament of individuals.

Methods This research is an applied study in term of aims and it has done by a descriptive-survey method. In this article, we examine the relationship between Temperament and color of environmental elements. To investigate the hypothesis, a survey was conducted on 144 housewives in one of the settlements of Kerman. In this study, data were collected through a medical questionnaire of Temperament and field observation of residential units and recording the colors of elements such as walls, ceilings. Data analyszed using the Chi-square test in SPSS V. 22 software.

Results The results showed that there is a significant relationship between the Temperaments of individuals and the colors of walls, In terms of Warmth and coldness $(P<0.001)$. Also, the results of the test show that there is no significant relationship between the ceilings color with the Temperament of the test subjects. $(P=0.517,0.124,0.115 \& 0.137$, respectively)

Conclusion It seems that there is a significant relationship between the Temperament and the colors used in the environment and the Temperaments of the individuals affected by the color of the surrounding environment. Therefore, architects as the main designers of the building, in collaboration with traditional medicine practitioners with color Temperament knowledge can design interior design and home decoration that enhance the health of users.

\section{Extended Abstract}

\section{Introduction}

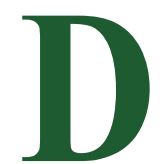

ue to its relationship with mankind, architecture is critical. Thus, it is essential to address the relationship between the individuals and the environment. The perception and experience of space in architecture is mainly focused on quality; several variables are involved in the quality of this experience, the interaction of which indicates the resulting experience. The basis of the perception of the relationship between individuals and the environment and the quality of such interaction arises from the interaction of environmental components. This is because the quality of the living environment is considered in various sciences, like medicine.

\section{* Corresponding Author:}

Vahideh Rahimi Mehr, PhD.

Address: Department of Architecture, Faculty of Art, Architecture \& Urbanism, Kerman Branch, Islamic Azad University, Kerman, Iran. Tel: +98 (913) 1986371

E-mail: yekta.rahimi@yahoo.com 
From the perspective of medicine, environmental characteristics could affect the human brain and through the brain, impact other body parts. In this regard, color and light are among the environmental factors with historical effects on humans. They are both of the main features in architecture and are in our control more than any environmental dimension. Traditional Iranian medicine pays attention to human beings, as the most advanced creatures; it considers the human nature and temperament, and the human-environment interaction plays a strategic role in this area. This science filed is related to architecture in terms of considering the quality of the living environment.

According to the theories of traditional medicine, individuals' temperament changes under the influence of various factors; accordingly, their health is affected. Light and color are the most significant pillars of a healing environment. Therefore, the interaction of architectural knowledge with traditional medicine in the field of light and color of the environment could help improve the quality of human life. In traditional Iranian medicine, special attention has been paid to the physical characteristics of the natural and artificial environment and its role on human health. In the addressed environmental issues by this field, color has been of particular importance. This is because color is among the factors affecting health and treatment. Recognizing pain from a specific color to selecting a medication in a specific color and the method of treatment and maintenance of the patient in a specific color environment, indicate the attention of traditional medicine to color. Despite all the emphases of traditional Iranian medicine on the effect of color on maintaining health and treatment, the relationship between color and temperament remains undiscovered.

\section{Materials and Methods}

In terms of the purpose, this was an applied research, i.e., conducted by cross-sectional-analytical method in Kerman City, Iran in the Spring of 2017. This study explored the relationships between individuals' temperament and the color of the walls and ceiling of an artificial environment. The statistical population of the study included residential houses and housewives aged 20-40 years without a specific disease history. To investigate the hypothesis, research was conducted on 144 housewives and their homes in residential units in one of the towns of Kerman.

In this study, data related to the temperament of individuals were collected through a medical questionnaire to determine the temperament and field observation of residential units, as well as recording the colors of the walls, ceiling of the bedroom, and living room. Before visiting the houses, a checklist, consisting of two columns of space and the color of the environment was prepared to record field observations in relation to the color of the wall and ceiling during the visit. To complete the checklist, under the color column of the environment, the colors precepted from the observation of the space were placed; according to the temperament of each color, the code related to that temperament was registered. To determine the temperament of colors, the law of color composition and construction was employed; accordingly, due to the specificity of multi-color temperament in medical texts, the temperament of other colors observed in the environment was obtained.

In addition, Chi-squared test was used to investigate the relationship between the color variables of environmental elements and temperament. The results of Chi-squared test suggested the P-value of the relationship between variables. If the results of the study of the relationship between variables were less than $5 \%$, it could be concluded that the relationship is significant.

\section{Results}

The results of statistical data analysis indicated that in general, there was a significant relationship between the temperament of individuals and the temperament of environmental elements. There was a significant relationship between human temperament and the temperament of the color of the bedroom and living room walls, in terms of warmth, coldness, dryness, and wetness $(\mathrm{P}<0.001)$. The obtained data revealed no significant relationship between the color of the bedroom and living room ceilings and the temperament of individuals in terms of warmth, coldness, and dryness $(\mathrm{P}=0.517, \mathrm{P}=0.124, \mathrm{P}=0.115 \& \mathrm{P}=0.137$, respectively).

\section{Discussion}

There seems to exist a significant relationship between an individual's temperament and the colors used in the environment. Moreover, individuals' temperament is influenced by the color of the environment. The colors of the environment of a place affect the mood of individuals; thus, impact their health. In other words, the environment and environmental elements, due to their special nature and temperament, affect the human nature and temperament. Furthermore, since these elements are the source of actions, they impose a specific state on the human body and mind. If this condition agrees with modifying the individual's temperament, it plays a role in maintaining his/her health, and if it adversely affects the person's temperament, it causes one of the disorders to prevail and endangers the person's health. 


\section{Conclusion}

Therefore, due to the multiple subtleties in the design of the environment, architects, as the main designers of the building, with the cooperation of traditional medicine practitioners, and with awareness about the color temperament in interior design, could develop an environment to promote the health of users to create the desired living environment. It is an effective component in guiding individuals towards peace.

\section{Ethical Considerations}

Compliance with ethical guidelines

All ethical principles are considered in this article.

Funding

This research did not receive any grant from funding agencies in the public, commercial, or non-profit sectors.

Authors' contributions

All authors were equally contributed in preparing this article.

Conflicts of interest

The authors declared no conflict of interest.

Acknowledgements

The author appreciate Dr. Mehrzad Mehrabani and Dr. Heshmatollah Motadin. We also thank the former Deputy of Mayor of Kerman Province, Dr. Mohammad Reza Khani, and the residents of the town of "Noon and AlQalam" in Kerman City, particularly, the management, Mr. Borkhori, for their cooperation. 


\section{بررسى رابطه مزاج انسان با رنتى محيط: يك مطالعه مقطعى تحليلى در شهر كرمان (عوسا)}

'وحيده رحيمىمهر'

I. كروه معمارى، دانشكده هنر، معمارى و شهرسازي، واحد كرمان، دانشُّاه آزاد اسلامى، كرمان، ايران

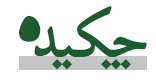

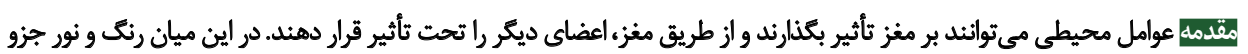

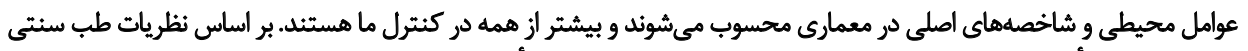

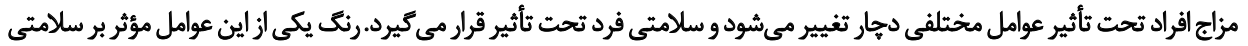

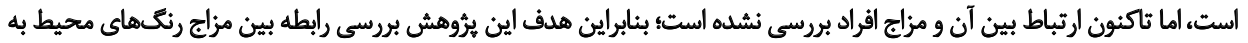

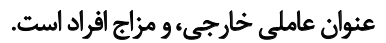

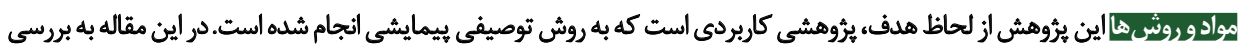

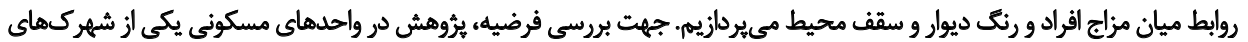

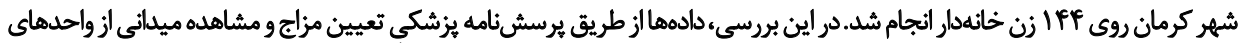

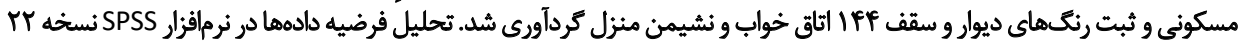

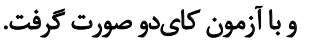

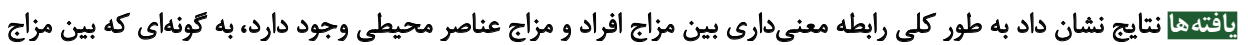

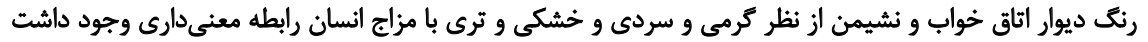

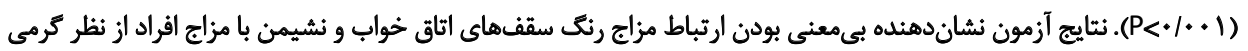

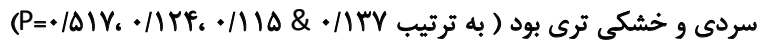

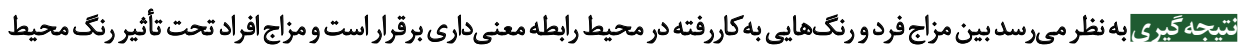

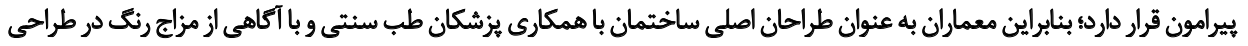

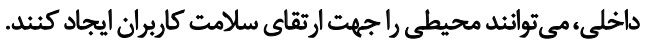

اطلاعات مقاله:

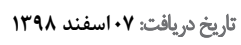

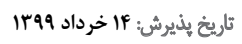
تاريخ انتشار: 11 تير 199

كليدوازوها:

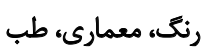
سنتى ايران، مزاج

ارتباط انسان و محيط، كاهي با انديشيدن، كاهي با لمس كان

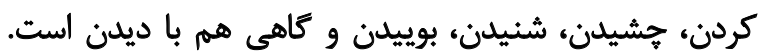

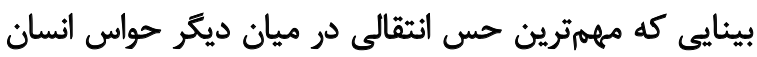

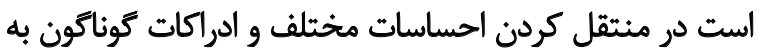

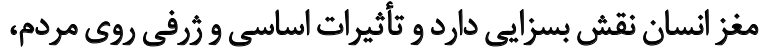

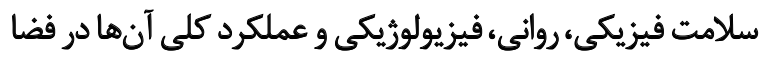

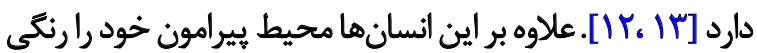

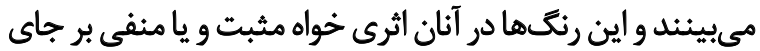

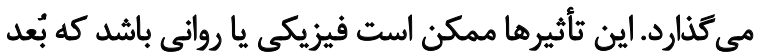

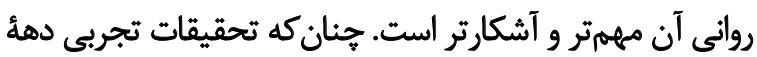

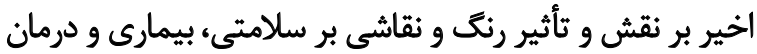

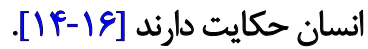
رنك عامل مهمى است كه بر عملكرد استفادهنئله از فضا،

زندكى در محيط با مبادله مداوم همراه است [1] و زندكى

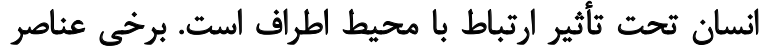

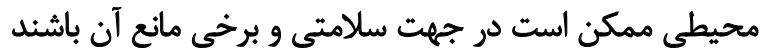

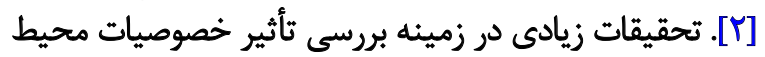

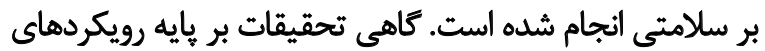

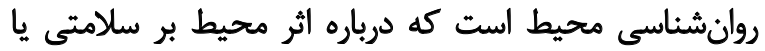

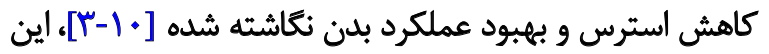

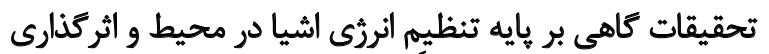

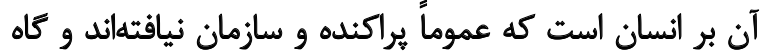

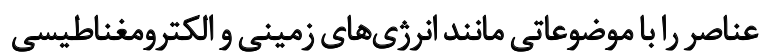

و انرزى عرمانى آميختهاند [11]

\section{-}

: نويسنده مسئول:

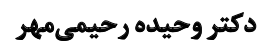

نشائى: كرمان، دانشكاه آزاد اسلامى، واحد كرمان، دانشكده هثر، معمارى و شهرسازي، كروه معمارى.

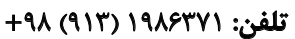
بست الكترونيكي: بلئ:yekta.rahimi@yahoo.com 
جدول ا.ارتباط رنى با امور طبيعى

\begin{tabular}{|c|c|c|c|}
\hline رنى & خلط & مزاج & اركان اربعه \\
\hline زرد & صفرا & كرم و خشك & آتش \\
\hline قرمز & دم & كرم وتر & هوا \\
\hline سفيد & بلغم & سرد و تر & آب \\
\hline سياه & سودا & سرد وخشك & خاى \\
\hline
\end{tabular}

روش همبستخى به بررسى ارتباط رنح عناصر محيطى به عنوان

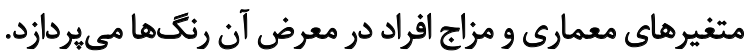

\section{مواد و روشها}

اين يُؤهش از لحاظ هدف كاربردى است كه به روش مقطعى

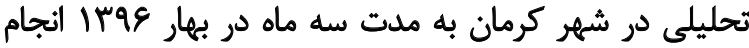

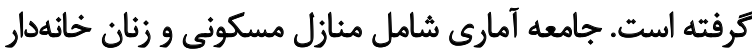

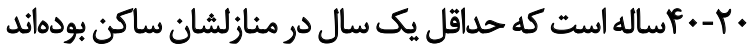

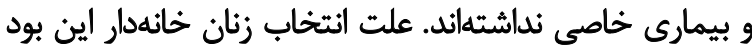

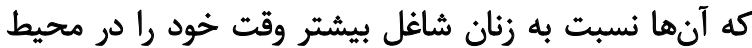

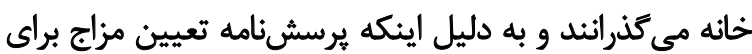

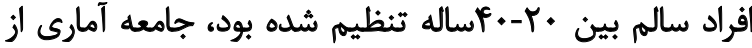

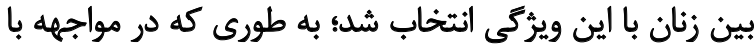

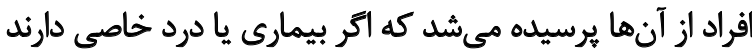

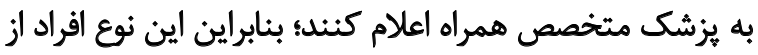

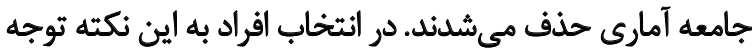

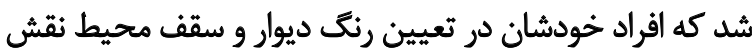

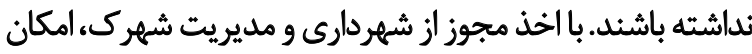

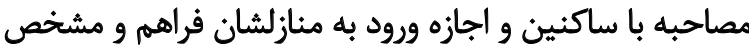

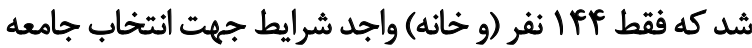

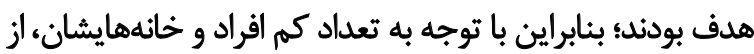

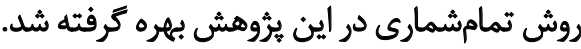

قبل از مراجعه به منازل جكىليستى آماده شد تا مشاهدات

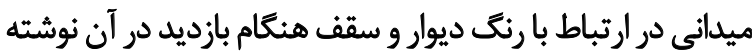

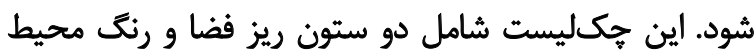

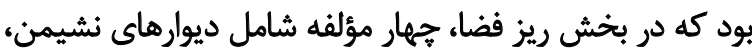

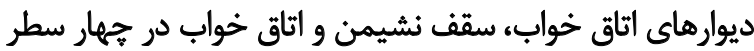

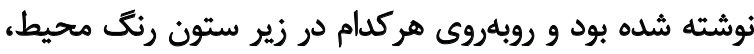

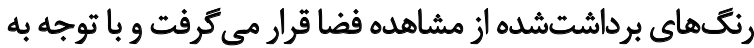

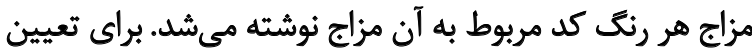

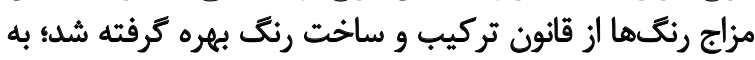

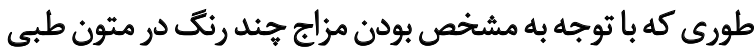

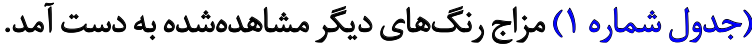
با توجه به برداشت ميدانى حاصل از بازديد منزل افراد، هنكام
تأثير مى گذارد و نقش مهمى در معمارى ايفا مى كند. جنان كهي

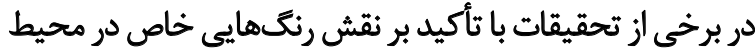

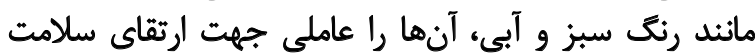

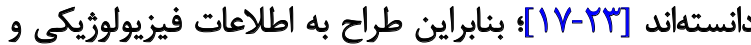

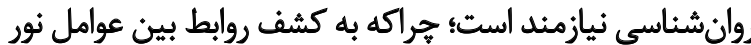

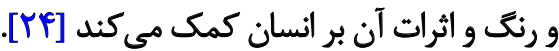

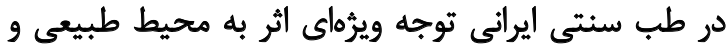

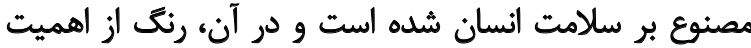

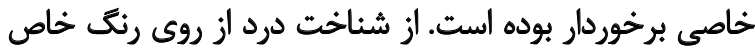

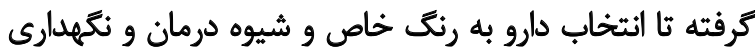

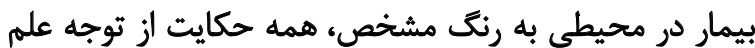

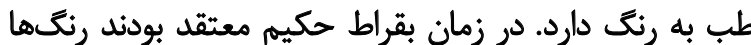

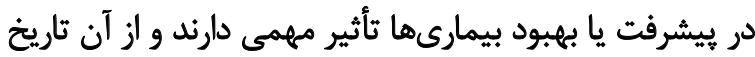

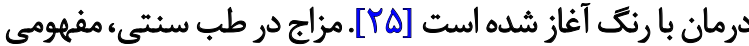

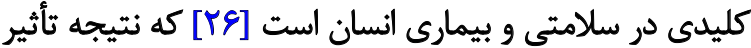

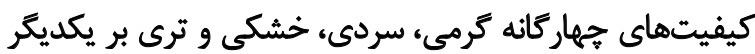

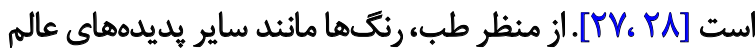

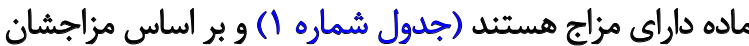

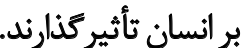

طب سنتى به طور كلى براى حفظ سلامتى، استفاده از تركيب

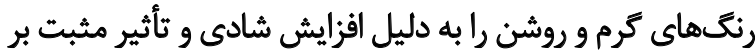

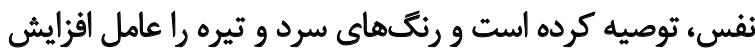

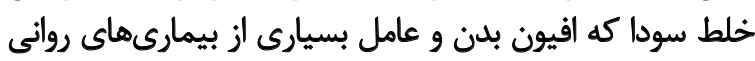

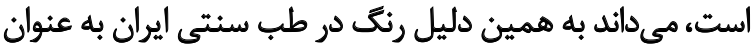

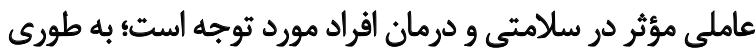

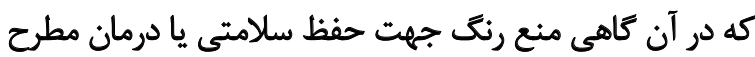

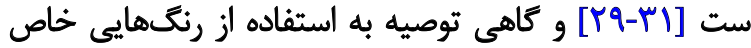

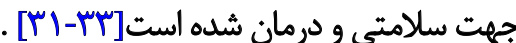

طبق نظريات طب سنتى مزاج افراد تحت تأثير رنغ محيط

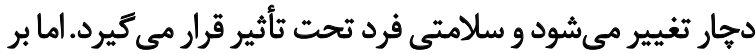

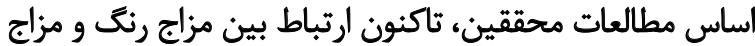

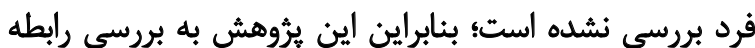

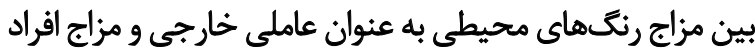
مي يردازد. اين تحقيق يك مطالعه توصيفى تحليلى است كه به به 
جدول r. توزيع فراوانى مزاج افراد شركت كنئده در بُروهش

\begin{tabular}{|c|c|c|}
\hline فراوانى (درصد) & & \\
\hline $8 \cdot(91 / M)$ & كرم & \multirow{4}{*}{ مزاج از نظر كرمي و سردى } \\
\hline$\Delta \Lambda(\varphi \cdot / \mu)$ & سرد & \\
\hline$r e(W \cdot)$ & معتل & \\
\hline $\operatorname{lif}(1+.1+)$ & جمع & \\
\hline$\varepsilon \Delta(F \Delta / 1)$ & خشك & \multirow{4}{*}{ مزاج از نظر خشكى و ترى } \\
\hline$\Delta 1(\Gamma \Delta / \varphi)$ & تر & \\
\hline$r(1 Q / \Delta)$ & معتل & \\
\hline $\operatorname{Iff}(1 . .1 *)$ & جمع & \\
\hline
\end{tabular}

به برداشت ميدانى و يرسشنامه مزاج وارد نرمافزار آمارى SPSS

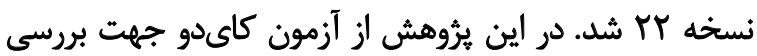

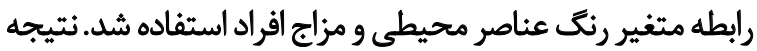

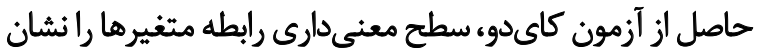

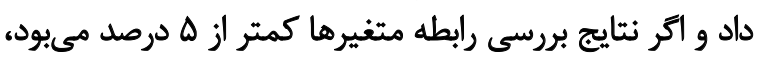
نتيجه كرفته مى شد كه رابطه موردنظر معنى دار است.

يافتهها

در يزؤهش حاضر |FF أخانه و زن خانهدار مورد بررسى قرار

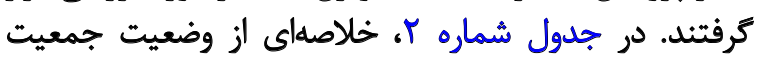
مشاركت كنندكان از نظر مزاج نشان داده شده است.

از آنجايى كه بر اساس مبانى نظرى، هر رنكَ داراى مزاج خاصى

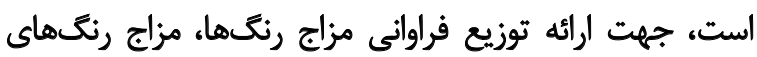

مراجعه، ابتدا براى تعيين مزاج زنان تحت آزمون، يرسشنامه

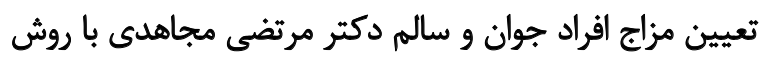

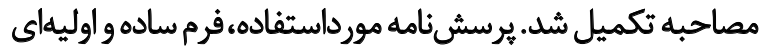

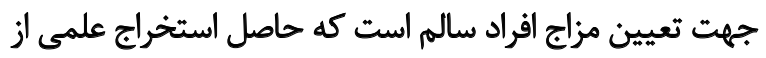

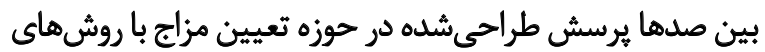

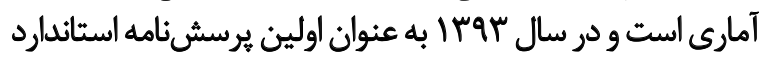

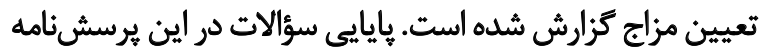

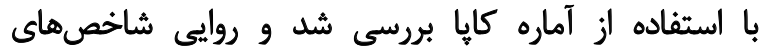

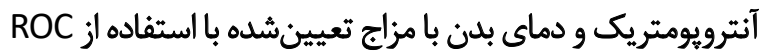

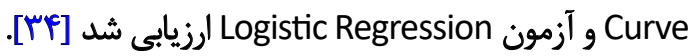
يس از تكميل يرسشنامه، مشاهدات ميدانى در جـكليستها

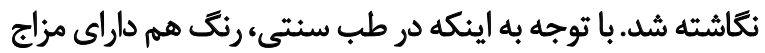

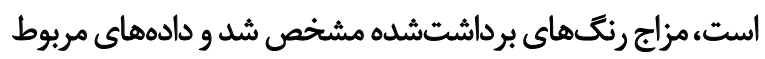

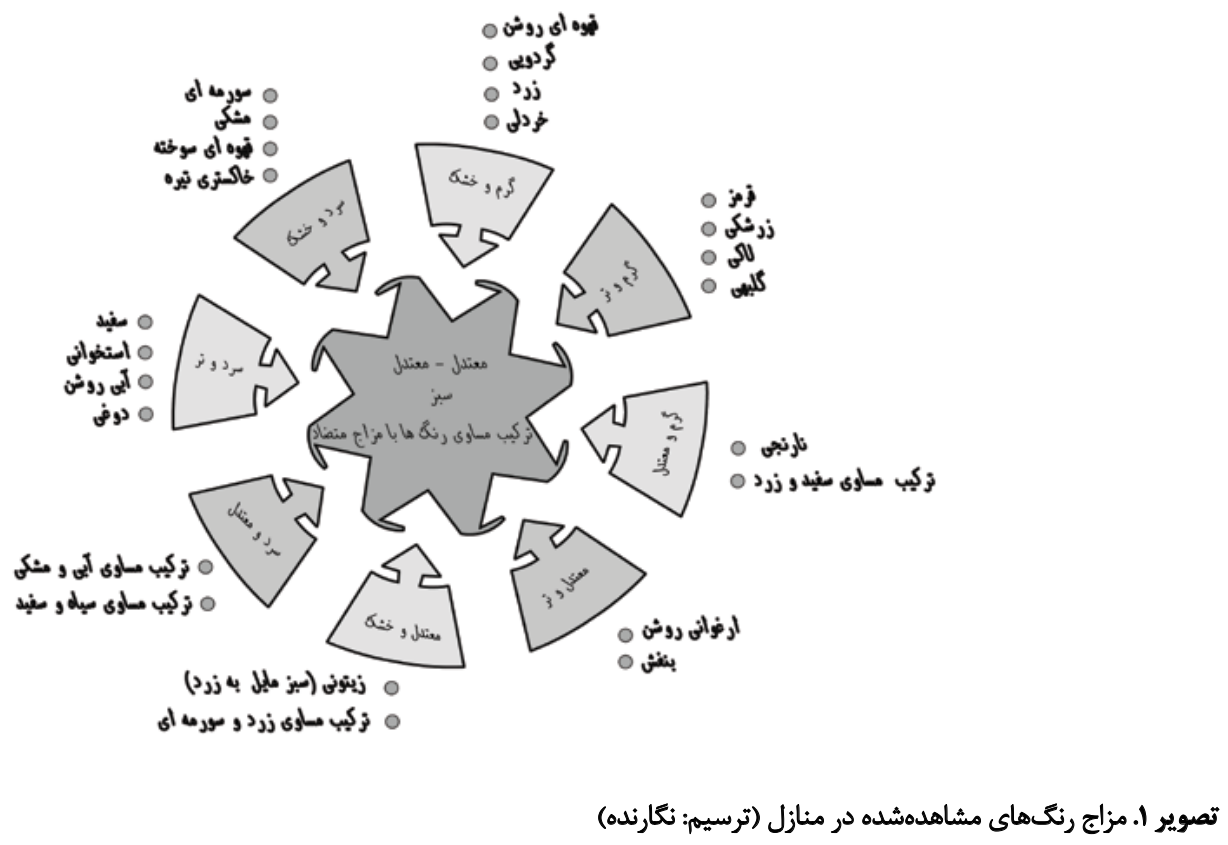


جدول ب. فراوائى رنكـها بر اساس گرمى و سردى مزاج

\begin{tabular}{|c|c|c|c|c|}
\hline \multicolumn{4}{|c|}{ فراوانى (درصد) } & \multirow{2}{*}{ رنّ عناصر و اجزا } \\
\hline جمع & معتدل & سرد & كرم & \\
\hline $\ln (1 . .1 \%)$ & $r \Delta(I V / P)$ & $r(\Delta \cdot / \cdot)$ & $P(T Y / S)$ & ديوار نشيمن \\
\hline $\operatorname{lff}(1 \cdots+1 \cdot)$ & $r A(19 / 4)$ & $\Delta q(F) / \cdot)$ & $\Delta V(r q / \varepsilon)$ & ديوار اثأق خواب \\
\hline $\operatorname{liff}(1 \cdot+1+)$ & $r \Lambda(19 / 4)$ & $\operatorname{Av}(\varepsilon \cdot / f)$ & $r q(r+/)$ & سقف نشيمن \\
\hline $\ln (1 . .1 \%)$ & $M(K / \Delta)$ & $U($ INI) & $r A(19 / 7)$ & سقف اتأ خواب \\
\hline
\end{tabular}

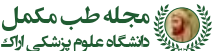

جدول F. فراوائى رنك ها براساس خشكى و ترى مزاج

\begin{tabular}{|c|c|c|c|c|}
\hline \multicolumn{4}{|c|}{ فراوانى (درصد) } & \multirow{2}{*}{ رنئ عناصر و اجزا } \\
\hline جمع & هعتدل & تر & خشيك & \\
\hline $\operatorname{liff}(1++1+)$ & $r \in(W I)$ & $n(f q / \mu)$ & $P V(r T / \varepsilon)$ & ديوار نشيمن \\
\hline $\ln (1 . .1)$. & $r \Lambda(19 / 4)$ & $r q(\Delta F / q)$ & $r V(r \Delta / V)$ & ديوار اثاث خواب \\
\hline $\ln (1 \ldots /)$. & $1 \cdot(8 / 9)$ & Irq (Aq/) & $\Delta(r / \Delta)$ & سقف نشيمن \\
\hline $\operatorname{lnp}(1 . .1 \%)$ & $1(* / V)$ & IfT (99/7) & - & سقف اتاقخواب \\
\hline
\end{tabular}

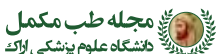

نشيمن و اتاق خواب به رنت كرم زندكى مى كنند، داراى مزاج

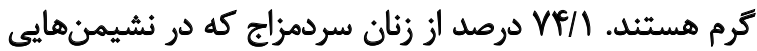

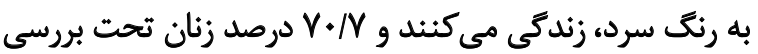

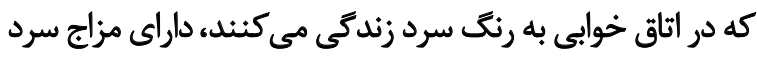

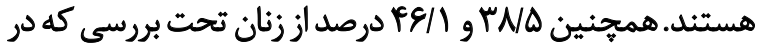

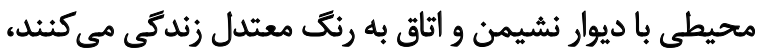

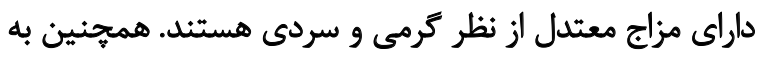

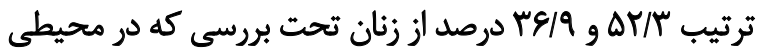

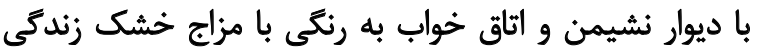

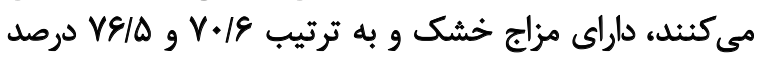

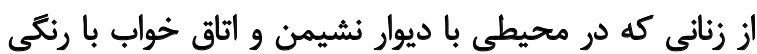

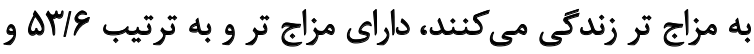

ثبتشده در Iff جكليست تكميلشده حاصل از مشاهدات ميدانى، در تصوير شماره إنشان داده شدهاند.

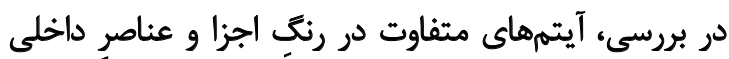

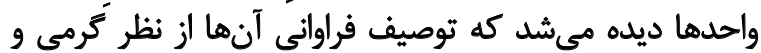

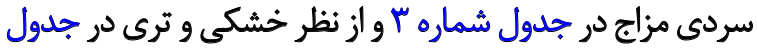

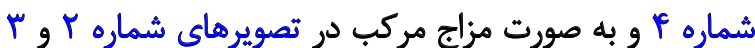
آورده شده است.

بررسى رابطه مزٔج فرد و رئَ ديوار

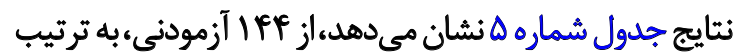

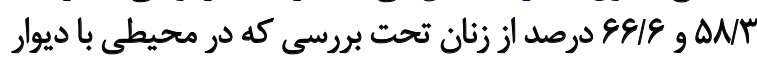

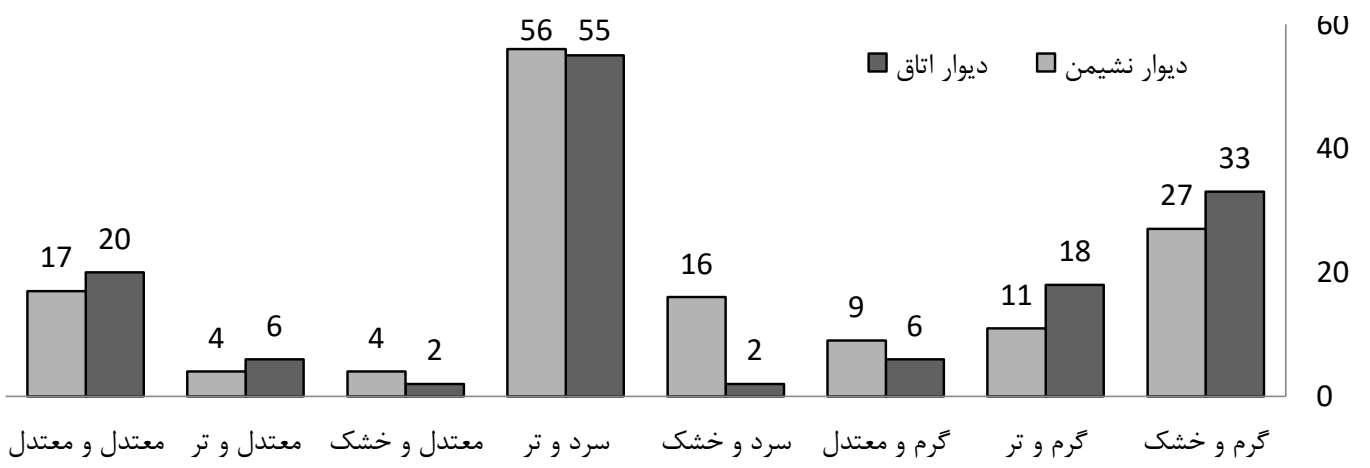




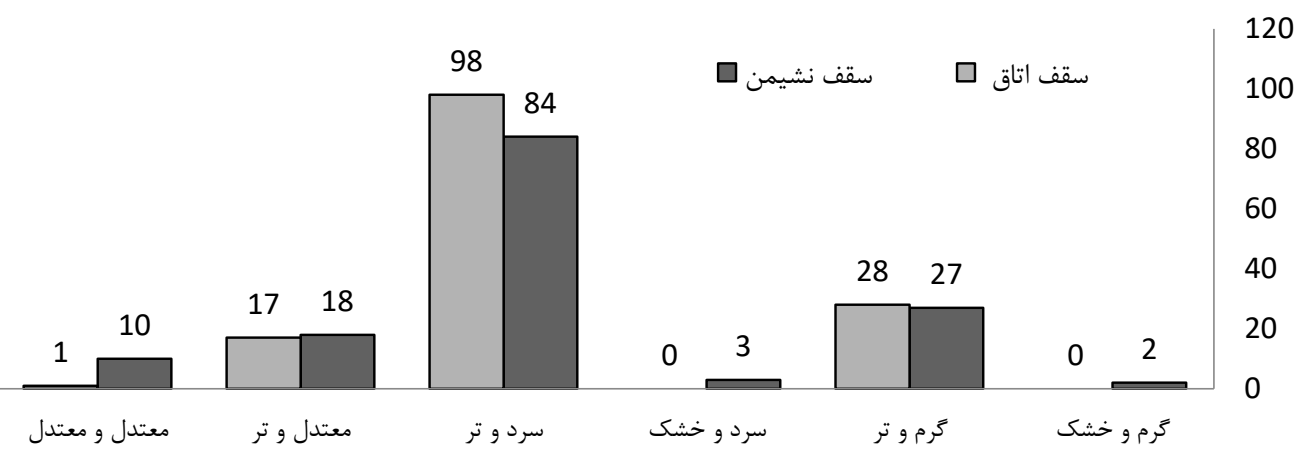

درصد از زنان تحت بررسى كه در محيطى با سقف نشيمن و و

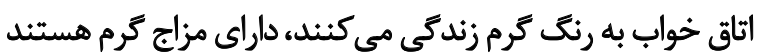

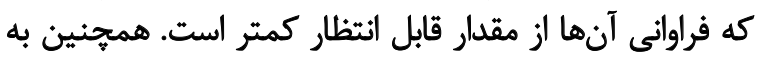
ترتيب

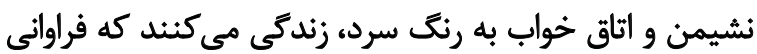

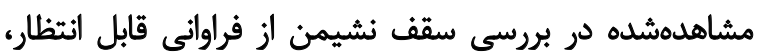

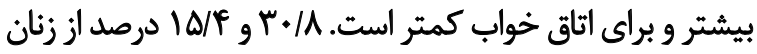

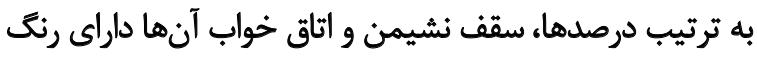

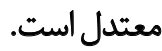

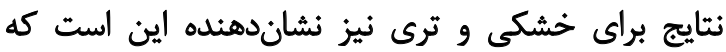

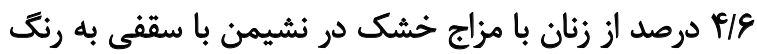

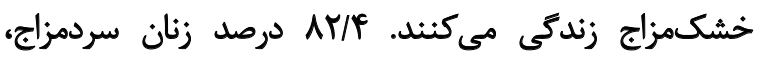

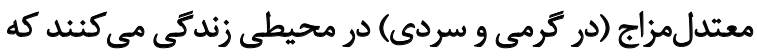

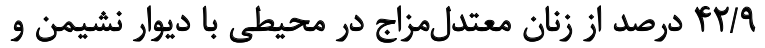

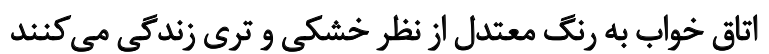

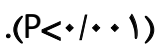

نتايج آزمون كاىدو (جدول شماره \&)، رابطه كاملأ معنادارى

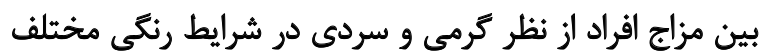

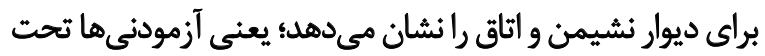

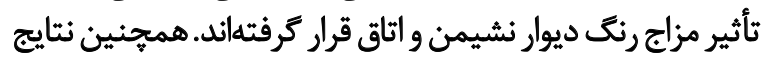

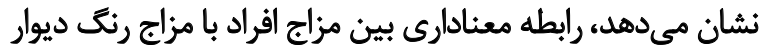

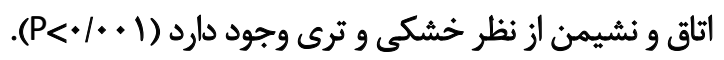
بروسي مزاج فردو ورنّى سقف

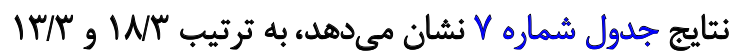

جدول هـ جدول فراوانى هاى مشاهدهشده مزاج افراد بر حسب رنك ديوار

\begin{tabular}{|c|c|c|c|c|c|c|c|c|c|}
\hline \multirow{2}{*}{\multicolumn{3}{|c|}{ مزاج رنك ديوار }} & \multirow[t]{3}{*}{ مزاج فرد } & \multirow{2}{*}{\multicolumn{3}{|c|}{ مزاج رنى ديوار }} & \multirow[t]{3}{*}{ مزاج فرد } & \multirow{3}{*}{ موارد مورد بررسى } & \multirow{3}{*}{ اجزا| } \\
\hline & & & & & & & & & \\
\hline معتدل & تو & خشك & & معتدل & سرد & كرم & & & \\
\hline 8 & ra & $m$ & \multirow{2}{*}{ خشك } & $r$ & $M$ & $r \Delta$ & \multirow{2}{*}{ كرم } & مقدار مشاهدهشده & \multirow{6}{*}{$\frac{c}{d}$} \\
\hline $9 / r$ & HNA & $\Delta r / r$ & & $11 / N$ & $r \%$ & $\Delta N /$ & & درصد & \\
\hline$\Delta$ & ع & 1. & \multirow[b]{2}{*}{ تر } & $\wedge$ & $\mu$ & r & \multirow[b]{2}{*}{ سرد سر } & مقدار مشاهدهده & \\
\hline $9 / 1$ & $V \cdot / 8$ & $19 / 8$ & & $\mid r / \lambda$ & $n \neq / 1$ & $|r /|$ & & درصد & \\
\hline 10 & 1. & $r$ & \multirow{2}{*}{ معتل } & 1. & 11 & $\Delta$ & \multirow{2}{*}{ معتل } & مقدار مشاهدهشه & \\
\hline$\Delta r / \&$ & $r \Delta / v$ & $1 . N$ & & rND & $P T / T$ & $19 / \pi$ & & درصد & \\
\hline$\wedge$ & $\pi$ & re & \multirow[b]{2}{*}{ خشك } & 1. & 1. & r. & \multirow[b]{2}{*}{ 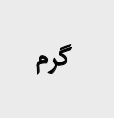 } & مقدار مشاهذهشده & \multirow{6}{*}{ 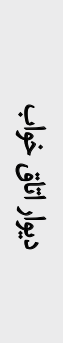 } \\
\hline$I r / T$ & $\Delta \cdot / A$ & $r e / q$ & & $19 / N$ & $19 N$ & $98 / 9$ & & & \\
\hline$\wedge$ & rq & $\varphi$ & \multirow[b]{2}{*}{ تر } & 8 & il & 11 & \multirow[b]{2}{*}{ سرد س } & مقدار مشاهلدشده & \\
\hline $1 ه / N$ & $v e / \Delta$ & $V / A$ & & $1 . / \pi$ & $V \cdot N$ & 19. & & درصد & \\
\hline ir & $\checkmark$ & 9 & \multirow[t]{2}{*}{ معتل } & ir & $\wedge$ & 8 & \multirow{2}{*}{ معتل } & مقدلر مشاهدمشده & \\
\hline$r T / q$ & $r \Delta /$. & $r r / 1$ & & 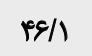 & $r \cdot / \Lambda$ & 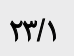 & & درصد & \\
\hline
\end{tabular}

(1) 
جدول \&. آزمون نيكويى برازش كايدو براى بررسى رابطه دو متغير مزاج اقراد و رنك ديوار

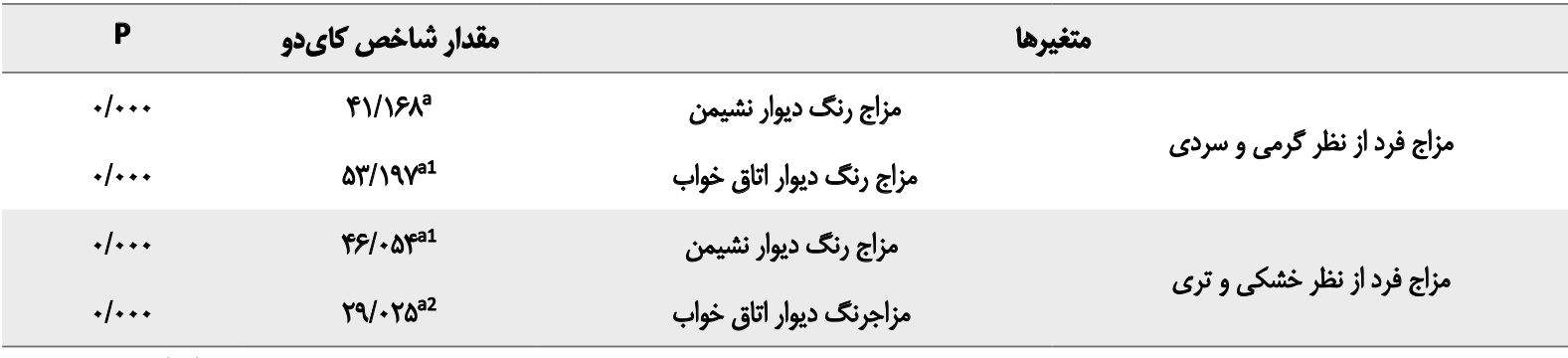

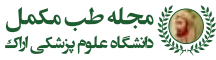

a: 1 cells $(11.1 \%)$ have expected count less than 5 . The minimum expected count is 4.51 ;

a1: 0 cells $(0.0 \%)$ have expected count less than 5 . The minimum expected count is 5.06 ; a2: 0 cells $(0.0 \%)$ have expected count less than 5 . The minimum expected count is 5.44 .

جدول V. جدول فراواني هاى مشاهدهده مزاج اقراد بر حسب رنك سقف

\begin{tabular}{|c|c|c|c|c|c|c|c|c|c|}
\hline \multicolumn{3}{|c|}{ مزاج رنئى سقف } & \multirow{3}{*}{ مزأج فرد } & \multicolumn{3}{|c|}{ مزاج رنئَ سقف } & \multirow{3}{*}{ فزاج } & \multirow{3}{*}{ موارد مورد بررسى } & \multirow{3}{*}{ اجزا } \\
\hline \multicolumn{3}{|c|}{ دوصد } & & & درصد & & & & \\
\hline معتدل & تو & خشك & & معتدل & سرد & كرم & & & \\
\hline r & \&. & $r$ & \multirow{2}{*}{ خشك } & $\wedge$ & il & 11 & \multirow{2}{*}{ كرم } & مقدار مشاهدهشده & \multirow{6}{*}{$\begin{array}{l}\xi \\
\xi \\
\xi \\
\xi \\
\xi\end{array}$} \\
\hline$r / 1$ & $9 T / \pi$ & r/s & & $\mid r / \%$ & ENF & Wr & & درهد & \\
\hline$\checkmark$ & RT & r & \multirow[b]{2}{*}{ تز } & ir & 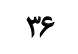 & 1. & \multirow[b]{2}{*}{ سرد سرد } & مقدار مشاهلهشده & \\
\hline $1 w / N$ & $A r / F$ & $r / q$ & & $r \cdot N$ & $e r / 1$ & $I V / T$ & & درهد & \\
\hline 1 & rV & - & \multirow{2}{*}{ معتل م م } & $\wedge$ & 1. & $\wedge$ & \multirow{2}{*}{ معتل } & مقلار مشاهلدشده & \\
\hline$r / s$ & $q s / 4$ & $\%$ & & $r \cdot / \Lambda$ & HNA & $r+/ s$ & & درصد & \\
\hline • & \&Q & - & \multirow{2}{*}{ خشك } & $\wedge$ & pr & $\wedge$ & \multirow{2}{*}{ كرم } & مقدار مشاهدهشده & \multirow{6}{*}{$\begin{array}{l}\frac{c}{b} \\
C_{i} \\
\frac{c_{i}}{\varepsilon_{i}} \\
\xi_{i}\end{array}$} \\
\hline$\%$ & 1.01 & - & & $\mid r / r$ & $V^{\prime} / \mathrm{F}$ & $\mid r / \pi$ & & درصد & \\
\hline - & ه) & - & \multirow{2}{*}{ تر } & 8 & mq & ir & \multirow{2}{*}{ سرد ل } & هقدار مشاهدهشده & \\
\hline .1 & $1 . . \%$ & - & & $1 \cdot / 6$ & $E V / T$ & $M / P$ & & درصد & \\
\hline 1 & Tr & - & \multirow{2}{*}{ هعتل } & f & 10 & $\checkmark$ & \multirow{2}{*}{ هest } & مقدار مشاهدهشده & \\
\hline$r / s$ & $q 8 / 5$ & - & & $10 / 6$ & $\Delta Y N$ & $r e / 9$ & & درول & \\
\hline
\end{tabular}

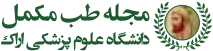

كرمى و سردى قرار نكرفتهاند. در مورد خشكى و ترى نيز رابطه

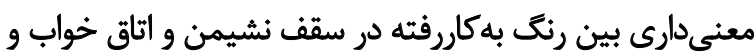

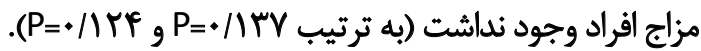

\section{بحث}

در يروهشهاى بيشين تأثير طراحى محيط و ويثزى هاى

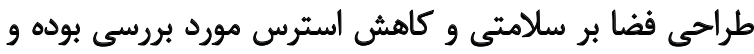

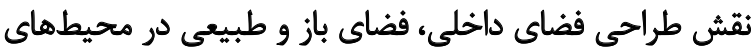

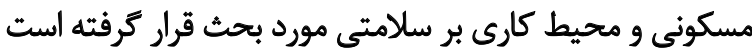

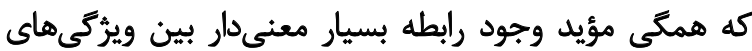

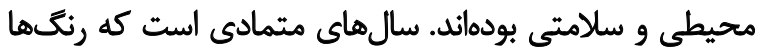

نشيمن خانههايشان داراي سقف با رنت ترمزاج است كه ميزان

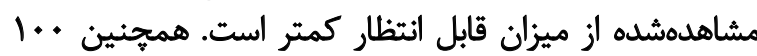

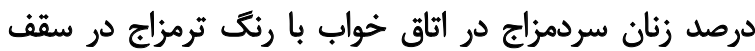

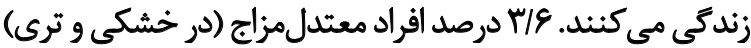

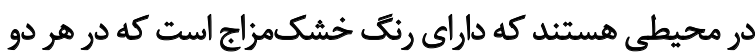
مورد، ميزان مشاهدهده از ميزان قابل انتظار كمتر است نتايج آزمون كايدو (جدول شماره ᄉ)، عدم وجود رابطه

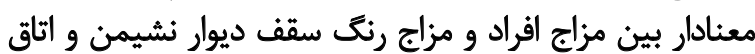

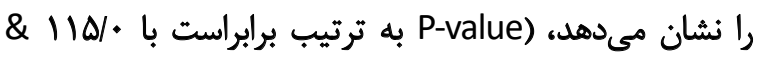

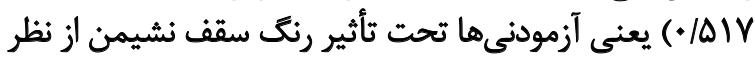


جدول A. آزمون نيكويى برارزش كايدو براى بررسى رابطه دو متغير مزاج افراد و رنك سقف

\begin{tabular}{|c|c|c|c|}
\hline $\mathbf{P}$ & مقدار شاخص كايدو & & \\
\hline $\begin{array}{l}.1110 \\
+1018\end{array}$ & $\begin{array}{l}\text { V/RTfa } \\
\text { m/MFYa2 }\end{array}$ & مزاج رنك سقف سقف نثيمن & هزاج فرد از نظلر كرمى و سردى \\
\hline $\begin{array}{l}. / 1 H Y \\
\text { IMY }\end{array}$ & $\begin{array}{l}8 / 4 x^{+a 1} \\
4 / / r^{\text {a3 }}\end{array}$ & مزاج رنك رنى سقف نشيمن & مزاج فرد از نظر خشكى و ترى \\
\hline
\end{tabular}

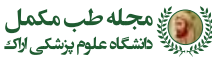

a:1 cells (11.1\%) have expected count less than 5 . The minimum expected count is 3.25 ;

a1: 0 cells $(.0 \%)$ have expected count less than 5 . The minimum expected count is 5.06 ;

a2: 6 cells $(66.7 \%)$ have expected count less than 5 . The minimum expected count is .97;

a3: 3 cells $(50.0 \%)$ have expected count less than 5 . The minimum expected count is .19 .

مى كند [Fr] ] با نتايج حاصل از تحقيق بايد عامل هشتم مزاج فرد

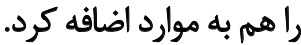

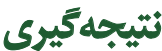

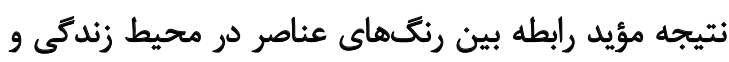

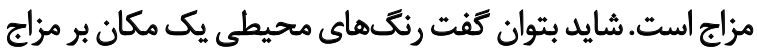

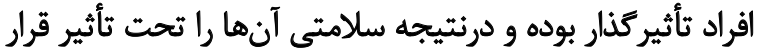

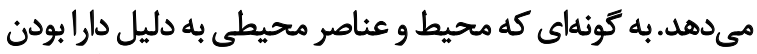

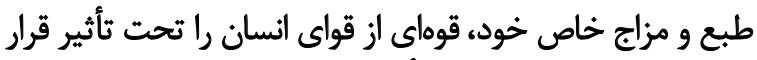

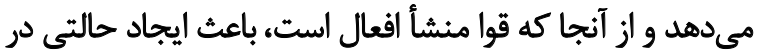

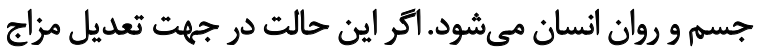

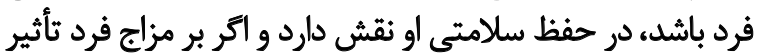

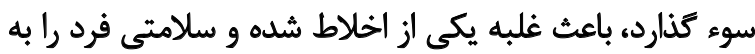

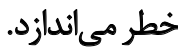

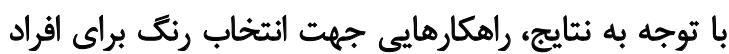

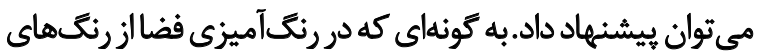

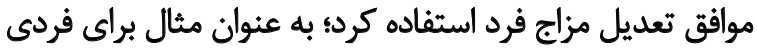

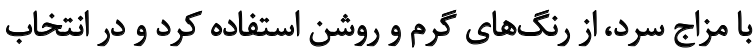

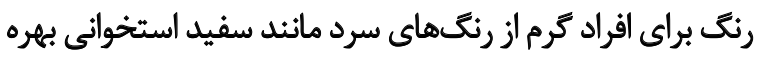

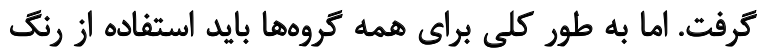

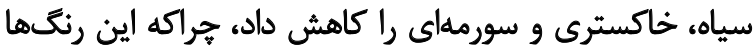

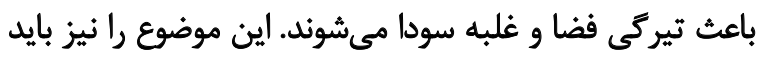

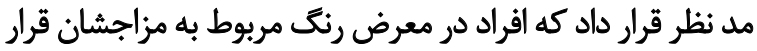

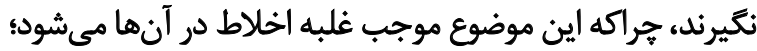

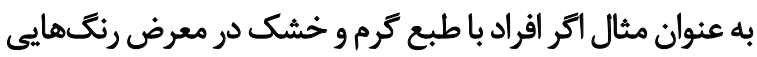

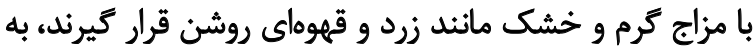

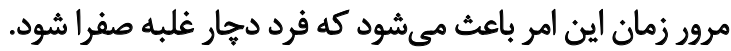

آثار خود را برذهن، رفتار، تعادل فكرى و جسمى، در ايجادي

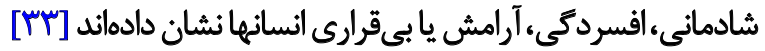

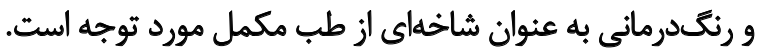

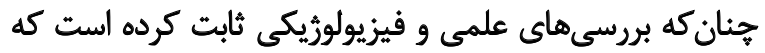

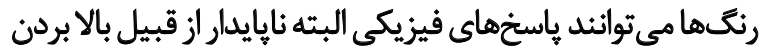

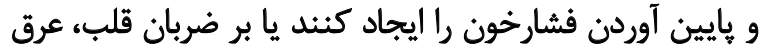

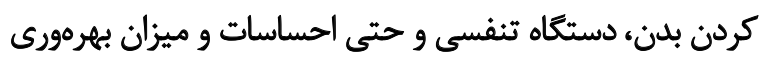

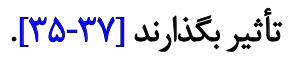

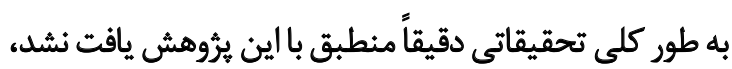

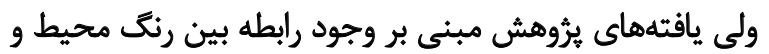

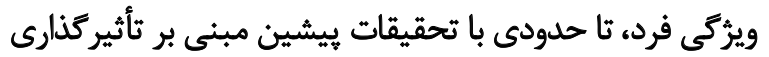

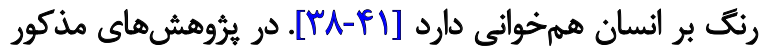

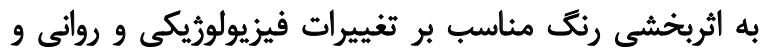

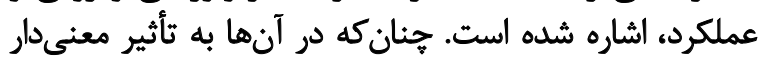

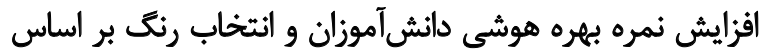

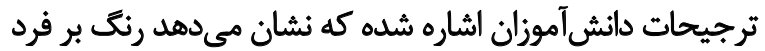
تأثير تذار بوده است.

نتايج آزمون كايدو در اين يُروهش نشان داد مزاج ديوار از نظر

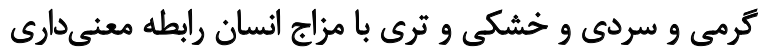

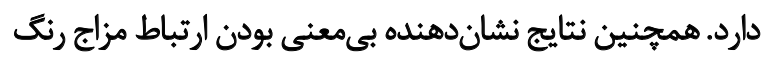

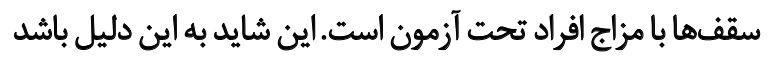

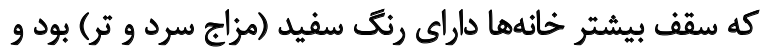

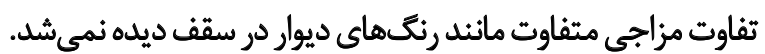

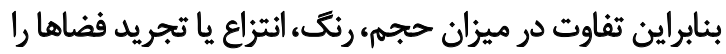

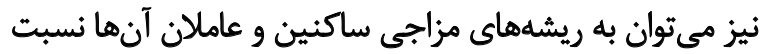

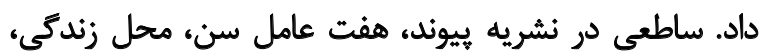

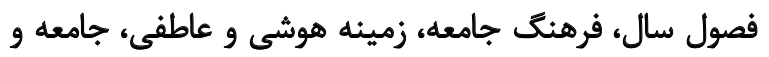

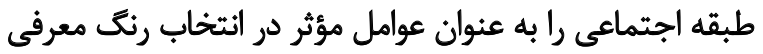




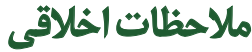

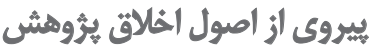

كليهى اصول اخلاقى در اين مقاله رعايت شده است

$$
\text { ماهى مالى }
$$

مقاله حاضر تحريرى تازه از رساله دكترى نكارنده با عنوان

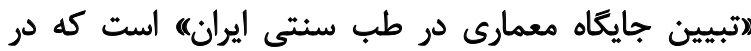

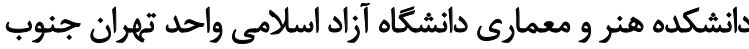

$$
\text { مشار كت ثويسند مكان }
$$

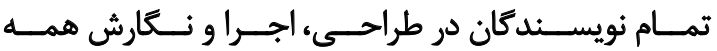

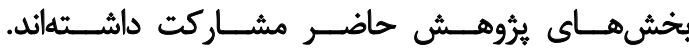

$$
\text { تعارض مناقع }
$$

بنابر اظهار نويسندكان، اين مقاله تعارض منافع ندارد.

$$
\text { تشكر و قدرداثي }
$$

لازم ميدانم از زحمات استادان بزركوار سركار خانم دكتر دئر

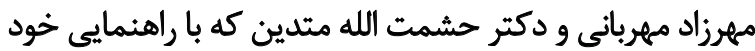

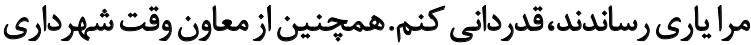

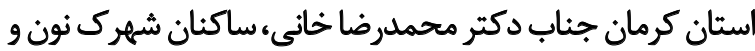

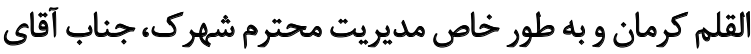
برخورى، به دليل همكارى و هماهنكى هاى لازم، كمال تشكر ران راب 


\section{References}

[1] Isfahani MM. [Health principles (Persian)]. Tehran: Tandis; 2005.

[2] Schweitzer M, Gilpin L, Frampton S. Healing spaces: Elements of environmental design that make an impact on health. The Journal of Alternative and Complementary Medicine. 2004; 10(Suppl 1):S-71-S-83. [DOI:10.1089/acm.2004.10.S-71]

[3] Bilotta E, Evans GW. Environmental stress. In: Steg L, van Den Berg AE, de Groot JLM, editors. Environmental psychology. London: Wiley; 2013. https://research.wur.nl/en/publications/environmental-psychology-anintroduction

[4] DuBose J, MacAllister L, Hadi Kh, Sakallaris B. Exploring the concept of healing spaces. HERD: Health Environments Research \& Design Journal. 2018; 11(1):43-56. [DOI:10.1177/1937586716680567] [PMID]

[5] MacAllister L, Bellanti D, Sakallaris BR. Exploring inpatients' experiences of healing and healing spaces: A mixed methods study. Journal of Patient Experience. 2016; 3(4):119-30. [DOI:10.1177/2374373516676182] [PMID] [PMCID]

[6] Burnard MD, Kutnar A. Wood and human stress in the built indoor environment: A review. Wood Science and Technology. 2015; 49:969-86 [DOI:10.1007/s00226-015-0747-3]

[7] Peng Hui FK, Aye L. Occupational stress and workplace design. Buildings. 2018; 8(10):133. [DOI:10.3390/buildings8100133]

[8] Petros AK, Georgi JN. Landscape preference evaluation for hospital environmental design. Journal of Environmental Protection. 2011; 2(5):63947. [DOI:10.4236/jep.2011.25073]

[9] Barton J, Bragg R, Wood C, Pretty J, editors. Green exercise: Linking nature, health and well-being. New York: Routledge; 2016. [DOI:10.4324/9781315750941]

[10] Rose E. Encountering place: A psychoanalytic approach for understanding how therapeutic landscapes benefit health and wellbeing. Health \& Place. 2012; 18(6):1381-7. [DOI:10.1016/j.healthplace.2012.07.002] [PMID]

[11] Najafi Ghezeljeh T, Bahonar E, Haghani H. [Investigating the effect of listening to nature sounds on the level of consciousness and physiological indices of patients with traumatic coma (Persian)]. Complementary Medicine Journal. 2019; 9(2):3642-56. http://cmja.arakmu.ac.ir/article1-594-en.html

[12] The House Whisperer. Epigenetic architecture: The consciousness matrix of buildings - genetic architecture ${ }^{\circledR}$ [Internet]. 2020 [Updated 2020]. Availble from: https://www.thehousewhisperer.tv/architects-academy/

[13] Brusseau ML, Ramirez-Andreotta M, Pepper IL, Maximillian J. Environmental impacts on human health and well-being. In: Brusseau ML, Pepper IL, Gerba CP, editors. Environmental and Pollution Science. 3rd ed. Cambridge, MA: Academic Press; 2019. p. 477-99. [DOI:10.1016/ B978-0-12-814719-1.00026-4]

[14] Briki W, Majed L. Adaptive effects of seeing green environment on psychophysiological parameters. Frontiers in Psychology. 2019; 10:252. [DOI:10.3389/fpsyg.2019.00252] [PMID] [PMCID]

[15] Lengen C. The effects of colours, shapes and boundaries of landscapes on perception, emotion and mentalising processes promoting health and well-being. Health \& Place. 2015; 35:166-77. [DOI:10.1016/j.healthplace.2015.05.016] [PMID]

[16] Yildirim K, Akalin-Baskaya A, Hidayetoglu ML. Effects of indoor color on mood and cognitive performance. Building and Environment. 2007; 42(9):3233-40. [DOI:10.1016/j.buildenv.2006.07.037]
[17] Cusack P, Lankston L, Isles Ch. Impact of visual art in patient waiting rooms: Survey of patients attending a transplant clinic in Dumfries. JRSM Short Reports. 2010; 1(6):1-5. [DOI:10.1258/shorts.2010.010077] [PMID] [PMCID]

[18] Korpela K, Hartig T. Restorative qualities of favorite places. Journal of Environmental Psychology. 1996; 16(3):221-33. [DOI:10.1006/ jevp.1996.0018]

[19] Mitchell R, Popham F. Greenspace, urbanity and health: Relationships in England. Journal of Epidemiology \& Community Health. 2007 61(8):681-3. [DOI:10.1136/jech.2006.053553] [PMID] [PMCID]

[20] Richardson EA, Mitchell R. Gender differences in relationships between urban green space and health in the United Kingdom. Social Science \& Medicine. 2010; 71(3):568-75. [DOI:10.1016/j.socscimed.2010.04.015] [PMID]

[21] Foley R, Kistemann T. Blue space geographies: Enabling health in place. Health \& Place. 2015; 35:157-65. [DOI:10.1016/j.healthplace.2015.07.003] [PMID]

[22] Mitchell R. Is physical activity in natural environments better for mental health than physical activity in other environments? Social Science \& Medicine. 2013; 91:130-4. [DOI:10.1016/j.socscimed.2012.04.012] [PMID]

[23] Ulrich RS. Visual landscapes and psychological well-being. Landscape Research. 1979; 4(1):17-23. [DOI:10.1080/01426397908705892]

[24] Ulrich RS. View through a window may influence recovery from surgery. Science. 1984; 224(4647):420-1. [DOI:10.1126/science.6143402] [PMID]

[25] Itten J. The elements of color: A treatise on the color system of Johannes Itten, based on his book the art of color. New York: John Wiley \& Sons; 1970. https://books.google.com/books?id=ofvRhNBgoCoC\&dq

[26] Farzan N. [Color and nature (Persian)]. Tehran: Tehran Publications; 1997. http://opac.nlai.ir/opac-prod/bibliographic/552725

[27] Naseri M, Rezaeiazdeh H, Taheripanah T, Naseri V. [Temperament theory in the Iranian traditional medicine and variation in therapeutic responsiveness, based on pharmacogenetics (Persian)]. Journal of Islamic and Iranian Traditional Medicine. 2010; 1(3):237-42. https://www.sid.ir/ fa/journal/ViewPaper.aspx?ID=149496

[28] Jakson WA. A short guide to humoral medicine. Trends in Pharmacological Sciences. 2001; 22(9):487-9. [PMID] [DOI:10.1016/s01656147(00)01804-6]

[29] Kord Afshari GR, Mohammadi Chenari H, Esmaeili S. [Nutrition in Iranian \& Islamic medicine (Persian)]. 17 ${ }^{\text {th }}$ ed. Tehran: Nasle Nikan; 2011 http://opac.nlai.ir/opac-prod/bibliographic/1224418

[30] Kermani MK. [Dallakiyyeh letter (Resaleyeh Dallakiyyeh) (Persian)] Tehran: Research Institute for Islamic \& Complementary Medicine; 2008. http://opac.nlai.ir/opac-prod/bibliographic/3771283

[31] Avicenna. The canon of medicine [A. Sharafkandi, Persian trans.]. Vol. 1. 10th ed. Tehran: Soroush; 2011. http://opac.nlai.ir/opac-prod/bibliographic/2094175

[32] Avicenna. The canon of medicine [A. Sharafkandi, Persian trans.]. Vol. 3. 8th ed. Tehran: Soroush; 2011. http://opac.nlai.ir/opac-prod/bibliographic/2094175

[33] Al-Ghozvali AIA. [Views of the full moon in the homes of pleasure (Men matale al-badr fi manazel al-surur) (Arabic)]. Vol. 2. Cairo: Edarato al-Vatan Publications; 1882 
[34] Ruga W. A healing environment, by design. Modern Healthcare. 2000; 30(44):24. [PMID]

[35] Mojahedi M, Naseri M, Majdzadeh R, Keshavarz M, Ebadini M, Nazem $E$, et al. Reliability and validity assessment of Mizaj Questionnaire: A novel self-report scale in Iranian traditional medicine. Iranian Red Crescent Medical Journal. 2014; 16(3):e15924. [DOI:10.5812/ircmj.15924] [PMID] [PMCID]

[36] Kateb F. [Color in our age (Persian)]. In: Consulting Engineers of Herampey. Light, sound, color in art, architecture and urbanism. Tehran: Azarakhsh Press; 2015. http://opac.nlai.ir/opac-prod/bibliographic/3710943

[37] Savavibool N, Gatersleben B, Moorapun Ch. The effects of colour in work environment: A systematic review. Asian Journal of Behavioural Studies. 2018; 3(13):149-60. [DOI:10.21834/ajbes.v3i13.152]

[38] Elliot AJ, Maier MA, Moller AC, Friedman R, Meinhardt J. Color and psychological functioning: The effect of red on performance attainment. Journal of Experimental Psychology: General. 2007; 136(1):154-68. [DOI:10.1037/0096-3445.136.1.154] [PMID]

[39] Kurt S, Osueke KK. The effects of color on the moods of college students. SAGE Open. 2014; 4(1). [DOI:10.1177/2158244014525423.]

[40] Lengen C. The effects of colours, shapes and boundaries of landscapes on perception, emotion and mentalising processes promoting health and well-being. Health \& Place. 2015; 35:166-77. [DOI:10.1016/j.healthplace.2015.05.016] [PMID]

[41] Elliot AJ. Color and psychological functioning: A review of theoretical and empirical work. Frontiers in Psychology. 2015; 6:368. [DOI:10.3389/ fpsyg.2015.00368] [PMID] [PMCID]

[42] Sateei E. The effect of colors in human life. Journal of link. 1993; 52:120-3. https://www.noormags.ir/view/fa/articlepage/147349/ 
This Page Intentionally Left Blank 